\title{
Radio $k$-labeling of paths
}

\author{
Laxman Saha ${ }^{1}$ (D), Satyabrata Das ${ }^{1}$ (D), Kinkar Chandra Das $^{2 *}$ (D), \\ Kalishankar Tiwary ${ }^{3}$ (D) \\ ${ }^{1}$ Department of Mathematics, Balurghat College, Balurghat 733101, India \\ 2 Department of Mathematics, Sungkyunkwan University, Suwon 16419, Republic of Korea \\ ${ }^{3}$ Department of Mathematics, Raiganj University, Raiganj 733134, India
}

\begin{abstract}
The Channel Assignment Problem (CAP) is the problem of assigning channels (nonnegative integers) to the transmitters in an optimal way such that interference is avoided. The problem, often modeled as a labeling problem on the graph where vertices represent transmitters and edges indicate closeness of the transmitters. A radio $k$-labeling of graphs is a variation of CAP. For a simple connected graph $G=(V(G), E(G))$ and a positive integer $k$ with $1 \leq k \leq \operatorname{diam}(\mathrm{G})$, a radio $k$-labeling of $G$ is a mapping $f: V(G) \rightarrow\{0,1,2, \ldots\}$ such that $|f(u)-f(v)| \geq k+1-d(u, v)$ for each pair of distinct vertices $u$ and $v$ of $G$, where $\operatorname{diam}(\mathrm{G})$ is the diameter of $G$ and $d(u, v)$ is the distance between $u$ and $v$ in $G$. The span of a radio $k$-labeling $f$ is the largest integer assigned to a vertex of $G$. The radio $k$-chromatic number of $G$, denoted by $r c_{k}(G)$, is the minimum of spans of all possible radio $k$-labelings of $G$. This article presents the exact value of $r c_{k}\left(P_{n}\right)$ for even integer $k \in\left\{\left\lceil\frac{2(n-2)}{5}\right\rceil, \ldots, n-2\right\}$ and odd integer $k \in\left\{\left\lceil\frac{2 n+1}{7}\right\rceil, \ldots, n-1\right\}$, i.e., at least $65 \%$ cases the radio $k$-chromatic number of the path $P_{n}$ are obtain for fixed but arbitrary values of $n$. Also an improvement of existing lower bound of $r c_{k}\left(P_{n}\right)$ has been presented for all values of $k$.
\end{abstract}

Mathematics Subject Classification (2010). 05C12, 05C15, 05C78

Keywords. channel assignment, radio $k$-labeling, radio $k$-chromatic number, span

\section{Introduction}

The Channel Assignment Problem (CAP) is the problem of assigning channels (nonnegative integers) to the stations in an optimal way such that interference is avoided. CAP plays an important role in wireless network and a well-studied interesting problem. Many researchers have modeled CAP as an optimization problem as follows: Given a collection of transmitters to be assigned operating frequencies and a set of interference constraints on transmitter pairs, find an assignment that satisfies all the interference constraints and minimizes the value of a given objective function. In 1980, Hale [11] has modeled FAP as a Graph labeling problem (in particular as a generalized graph labeling problem) and is an active area of research now. Griggs and Yeh [10] concentrated on the fundamental case

\footnotetext{
*Corresponding Author.

Email addresses: laxman.iitkgp@gmail.com (L. Saha), sdas1012@gmail.com (S. Das), kinkardas2003@gmail.com (K.C. Das), tiwarykalishankar@yahoo.com (K. Tiwary)

Received: 01.06.2019; Accepted: 25.02.2020
} 
of $L(2,1)$-labelings. The $L(p, q)$-labeling problem $(p, q>0)$ and its variants have been studied extensively (see e.g. [1,2,7-12,14,20,29-32,34,35]).

Motivated by FM channel assignments, a new model, namely the radio $k$-labeling problem was introduced in $[4,15]$ and studied further in $[22,25,33]$. For a simple connected graph $G=(V(G), E(G))$ and a positive integer $k$ with $1 \leq k \leq \operatorname{diam}(\mathrm{G})$, a radio $k$-labeling of $G$ is a mapping $f: V(G) \rightarrow\{0,1,2, \ldots\}$ such that

$$
|f(u)-f(v)| \geq k+1-d(u, v)
$$

for each pair of distinct vertices $u$ and $v$ of $G$, where $\operatorname{diam}(\mathrm{G})$ is the diameter of $G$ and $d(u, v)$ is the shortest distance between $u$ and $v$ in $G$. The span of a radio $k$-labeling $f$, denoted by $\operatorname{span}_{f}(G)$, is the largest integer assigned to a vertex of $G$. The radio $k$ chromatic number of $G$, denoted by $r c_{k}(G)$, is the minimum of spans of all possible radio $k$-labelings of $G$. A radio $k$-labeling $f$ of $G$ is called minimal if $\operatorname{span}_{f}(G)=r n(G)$. Without loss of generality, for a minimal radio labeling $f$ we assume that $\min _{v \in V(G)} f(v)=0$, otherwise the span of $f$ can be reduced further by subtracting the positive integer $\min _{v \in V(G)} f(v)$ from all the labels of the vertices of the graph. For some specific values of $k$ there are specific names for radio $k$-labelings as well as the radio $k$-chromatic number in the literature, which are given in Table 1:

Table 1. Special names of radio $k$-labelings and radio $k$-chromatic number.

\begin{tabular}{ccc}
\hline$k$ & Name of labeling & $r c_{k}(G)$ \\
\hline 1 & Vertex coloring & Chromatic number, $\chi(G)$ \\
$\operatorname{diam}(G)$ & Radio & Radio number, $r n(G)$ \\
$\operatorname{diam}(G)-1$ & Antipodal & Antipodal number, $a c(G)$ \\
\hline
\end{tabular}

The radio $k$-labeling problem can be viewed as an instance of the $L\left(p_{1}, \ldots, p_{m}\right)$-labeling problem (see e.g. $[10,36]$ ), where $m, p_{1}, p_{2}, \ldots, p_{m} \geq 1$ are given integers, which aims at minimizing the span of a labeling $f: V(G) \rightarrow\{0,1,2, \ldots\}$ subject to $|f(u)-f(v)| \geq p_{i}$ whenever $d(u, v)=i, 1 \leq i \leq m$. In the special case where $m=k$ and $p_{i}=\max \{k+$ $1-i, 0\}$ for each $i$, the minimum span of such a labeling is exactly the radio $k$-chromatic number of $G$.

Determining the radio $k$-chromatic number of a graph is an interesting yet difficult combinatorial problem with potential applications to CAP. So far it has been explored for a few basic families of graphs and values of $k$ near to diameter. The radio number of any hypercube was determined in [16] by using generalized binary Gray codes. Ortiz et al. [27] have studied the radio number of generalized prism graphs and have computed the exact value of radio number for some specific types of generalized prism graphs. For two positive integers $m \geq 3$ and $n \geq 3$, the Toroidal grids $T_{m, n}$ are the cartesian product of cycle $C_{m}$ with cycle $C_{n}$. Morris et al. [26] have determined the radio number of $T_{n, n}$ and Saha et al. [30] have given exact value for radio number of $T_{m, n}$ when $m n \equiv 0(\bmod 2)$. The radio numbers of the square of paths and cycles were studied in [23,24]. For a cycle $C_{n}$, the radio number was determined by Liu and Zhu [25], and the antipodal number is known only for $n=1,2,3(\bmod 4)($ see $[3,13])$.

Surprisingly, even for paths finding the radio number was a challenging task. It is envisaged that in general determining the radio number would be difficult even for trees, despite a general lower bound for trees given in [22]. Till now, the radio number is known for very limited of families of trees. For path $P_{n}$, complete $m$-ary trees the exact values of radio number were determined in $[21,25]$. The results for path were generalized [25] to 
spiders, leading to the exact value of the radio number in certain special cases. In [28], Reddy et al. gave an upper bound for the radio number of some special type of trees. For an $n$-vertex path $P_{n}$, the exact value of $r c_{k}\left(P_{n}\right)$ is known only for $k=n-1$ [25], $n-2$ [16], $n-3$ [18], and $n-4$ ( $n$ odd) [19].

In literature, the exact value of $r c_{k}(G)$ are known only when $k \in\{\operatorname{diam}(\mathrm{G}), \operatorname{diam}(\mathrm{G})-1$, $\operatorname{diam}(G)-2\}$ and $G$ belong to some specific class of graphs. For path $P_{n}$, the radio $k-$ chromatic numbers $\left(r c_{k}\left(P_{n}\right)\right)$ are known for relatively more values of $k$, namely, $k=$ $n-1, n-2, n-3$ and $k=n-4$ (odd $n)$. This article presents the exact value of $r c_{k}\left(P_{n}\right)$ for even integer $k \in\left\{\left\lceil\frac{2(n-2)}{5}\right\rceil, \ldots, n-2\right\}$ and odd integer $k \in\left\{\left\lceil\frac{2 n+1}{7}\right\rceil, \ldots, n-1\right\}$, i.e., at least $65 \%$ cases the radio $k$-chromatic of the path $P_{n}$ are obtain for fixed but arbitrary values of $n$. Also an improvement of existing lower bound of $r c_{k}\left(P_{n}\right)$ has been presented for all values of $k$. In Table 2, we summarize the existing results and our results on $r c_{k}\left(P_{n}\right)$.

Table 2. Existing Results and Our Result on radio $k$-chromatic number of $P_{n}$. Here LB and UB denotes the lower and upper bounds for $r c_{k}\left(P_{n}\right)$.

\begin{tabular}{ccc}
\hline Author & Values of $k$ & $r c_{k}\left(P_{n}\right)$ \\
\hline Liu and Zhu [25] & $n-1$ & Exact value \\
Khennoufa and Togni [17] & $n-2$ & Exact value \\
Kola and Panigrahi [18] & $n-3$ & Exact value \\
Kola and Panigrahi [19] & $n-4$ (odd) & Exact value \\
Chartrand et al. [5] & $\leq n-3$ & LB and UB \\
Current article & $65 \%$ cases & Exact value \\
Current article & other cases & Improve LB \\
\hline
\end{tabular}

\section{Preliminaries}

Let $V\left(P_{n}\right)=\{0,1, \ldots, n-1\}$ be the vertex set of an $n$-vertex path $P_{n}$. The path $P_{n}$ has length $n-1$. For a fixed vertex $w \in V\left(P_{n}\right)$, level function is defined by $L_{w}(u)=d(w, u)$ for any $u \in V\left(P_{n}\right)$ and the weight of $P_{n}$ at $w$ is defined by $W_{P_{n}}(w)=\sum_{u \in V\left(P_{n}\right)} L_{w}(u)$. The weight $\omega\left(P_{n}\right)$ of $P_{n}$ is the smallest weight among all vertices of $P_{n}$, i.e., $\omega\left(P_{n}\right)=$ $\min \left\{W_{P_{n}}(w): w \in V\left(P_{n}\right)\right\}$. A vertex $C$ is said to be weight center of $P_{n}$ if $W_{P_{n}}(C)=$ $\omega\left(P_{n}\right)$.

Notation 2.1. We shall always fix a weight center $C$ for the path $P_{n}$. Then $P_{n} \backslash C$ consists of two branches (components), called the left and right branches of $P_{n} \backslash C$. The left branch and right branch of $P_{n}$ with respect to $C$ are denoted by $L\left(P_{n}\right)$ and $R\left(P_{n}\right)$, respectively. From here to onwards by $L(u)$ we mean $d(C, u)$ and called it the level of the vertex $u$ with respect to the weight center $C$. We denote the length of the common part of the paths from $C$ to $u$ and $C$ to $v$ by $\phi(u, v)$. Clearly, $\phi(u, v)=0$ if and only if $u$ and $v$ are in opposite sides of $C$.

Definition 2.2. For an $n$-vertex path $P_{n}$, by highest level vertex of $P_{n}$ we mean a vertex whose distance is maximum from a specified weight center $C$.

Observation 2.3. For an $n$-vertex path $P_{n}$ the following hold :

(1) If $C$ is the weight center of $P_{n}$, then $L\left(P_{n}\right)$ and $R\left(P_{n}\right)$ have maximum $\left\lfloor\frac{n}{2}\right\rfloor$ number of vertices.

(2) If $n$ is odd, then $P_{n}$ has exactly one weight center.

(3) If $n$ is even, then $P_{n}$ has two weight centers. 
Lemma 2.4. Let $P_{n}$ be a path of $n$ vertices with weight center at $C$. Then for distinct $u, v \in V\left(P_{n}\right)$ the following hold:

(1) $d(u, v)=L(u)+L(v)-2 \phi(u, v)$.

(2) $\phi(u, v)=0$ if and only if $C \in\{u, v\}$ or $u, v$ are in different branch.

(3) $\omega\left(P_{n}\right)= \begin{cases}\frac{n^{2}}{4} & \text { if } n \text { is even, } \\ \frac{n^{2}-1}{4} & \text { if } n \text { is odd. }\end{cases}$

In this article one target is to determine the radio $k$-chromatic number of path $P_{n}$. For this we need to determine the minimum span of a radio labeling of path $P_{n}$ in terms of some parameters like number of vertices, distances of minimum and maximum labeled (colored) vertices from the centroid. In Section 3, we discuss about the span of a radio labeling in terms of these parameters.

\section{Radio labeling of path}

Let $f$ be any radio labeling of $P_{n}$. So $f$ is injective and $f$ induces a linear order

$$
u_{0}, u_{1}, u_{2}, \ldots, u_{n-1}
$$

of the vertices of $P_{n}$ with $f\left(u_{0}\right)<f\left(u_{1}\right)<f\left(u_{2}\right)<\cdots<f\left(u_{n-1}\right)$. Clearly span of $f$ is $f\left(u_{n-1}\right)$. Now from the radio conditions we have the following for $0 \leq i \leq n-2$,

$$
f\left(u_{i+1}\right)-f\left(u_{i}\right) \geq n-d\left(u_{i}, u_{i+1}\right) .
$$

To make it an equality, we add a positive quantity $J_{f}\left(u_{i}, u_{i+1}\right)$, called jump of $f$ from $u_{i}$ to $u_{i+1}$, in right side of the inequality (3.2). Therefore,

$$
f\left(u_{i+1}\right)-f\left(u_{i}\right)=n-d\left(u_{i}, u_{i+1}\right)+J_{f}\left(u_{i}, u_{i+1}\right) .
$$

Summing up these $n-1$ equations, we get

$$
\begin{aligned}
f\left(u_{n-1}\right) & =\sum_{i=0}^{n-2}\left[f\left(u_{i+1}\right)-f\left(u_{i}\right)\right]+f\left(u_{0}\right) \\
& =\sum_{i=0}^{n-2}\left[n-d\left(u_{i}, u_{i+1}\right)+J_{f}\left(u_{i}, u_{i+1}\right)\right]+f\left(u_{0}\right) \\
& \geq n(n-1)-2 \sum_{i=0}^{n-1} L\left(u_{i}\right)+L\left(u_{0}\right)+L\left(u_{n-1}\right)+\sum_{i=0}^{n-2}\left[J_{f}\left(u_{i}, u_{i+1}\right)+2 \phi\left(u_{i}, u_{i+1}\right)\right] \\
& =n(n-1)-2 \omega\left(P_{n}\right)+f\left(u_{0}\right)+L\left(u_{0}\right)+L\left(u_{n-1}\right)+\sigma(f)
\end{aligned}
$$

where $\sigma(f)=\sum_{i=0}^{n-2} \sigma_{f}\left(u_{i}, u_{i+1}\right)$ and $\sigma_{f}\left(u_{i}, u_{i+1}\right)=J_{f}\left(u_{i}, u_{i+1}\right)+2 \phi\left(u_{i}, u_{i+1}\right)$. Here total jump $J(f)=\sum_{i=0}^{n-2} J_{f}\left(u_{i}, u_{i+1}\right)$. So the relationship between $\sigma(f)$ and $J(f)$ is $\sigma(f)=$ $J(f)+2 \sum_{i=0}^{n-2} \phi\left(u_{i}, u_{i+1}\right)$. If $u_{t}, u_{t+1}$ are in same branch then it is clear that $\sigma_{f}\left(u_{t}, u_{t+1}\right) \geq 2$.

Now we calculate the jumps from $u_{i}$ to $u_{i+1}$ and $u_{i+1}$ to $u_{i+2}$ under the following assumption:

Assumption 3.1. Vertices $u_{i}$ and $u_{i+2}$ are in the same branch of $P_{n}$ and vertex $u_{i+1}$ is in a different branch. 
Lemma 3.2. Let $u_{i}$ and $u_{i+2}$ be in the same branch of $P_{n}$ and let $u_{i+1}$ be in a different branch of $P_{n}$. Then

$$
J_{f}\left(u_{i}, u_{i+1}\right)+J_{f}\left(u_{i+1}, u_{i+2}\right) \geq \max \left\{2 L\left(u_{i+1}\right)+2 \phi\left(u_{i}, u_{i+2}\right)-n, 0\right\} .
$$

Proof. We have $f\left(u_{i+1}\right)-f\left(u_{i}\right)=n-d\left(u_{i}, u_{i+1}\right)+J_{f}\left(u_{i}, u_{i+1}\right)=n-L\left(u_{i}\right)-L\left(u_{i+1}\right)+$ $2 \phi\left(u_{i}, u_{i+1}\right)+J_{f}\left(u_{i}, u_{i+1}\right)$ and $f\left(u_{i+2}\right)-f\left(u_{i+1}\right)=n-d\left(u_{i+1}, u_{i+2}\right)+J_{f}\left(u_{i+1}, u_{i+2}\right)=$ $n-L\left(u_{i+1}\right)-L\left(u_{i+2}\right)+2 \phi\left(u_{i+1}, u_{i+2}\right)+J_{f}\left(u_{i+1}, u_{i+2}\right)$. Summing up we get

$$
f\left(u_{i+2}\right)-f\left(u_{i}\right)=2 n-L\left(u_{i}\right)-L\left(u_{i+2}\right)-2 L\left(u_{i+1}\right)+J_{f}\left(u_{i}, u_{i+1}\right)+J_{f}\left(u_{i+1}, u_{i+2}\right)
$$

where $J_{f}\left(u_{t}, u_{t+1}\right)=J_{f}\left(u_{t}, u_{t+1}\right)+2 \phi\left(u_{t}, u_{t+1}\right)$ for $t=i, i+1$. On the other hand, since $f$ is a radio labeling, we have

$$
f\left(u_{i+2}\right)-f\left(u_{i}\right) \geq n-d\left(u_{i}, u_{i+2}\right)=n-L\left(u_{i}\right)-L\left(u_{i+2}\right)+2 \phi\left(u_{i}, u_{i+2}\right) .
$$

Combining the two expressions above, we get

$$
J_{f}\left(u_{i}, u_{i+1}\right)+J_{f}\left(u_{i+1}, u_{i+2}\right) \geq 2 L\left(u_{i+1}\right)+2 \phi\left(u_{i}, u_{i+2}\right)-n .
$$

Since the value $J_{f}\left(u_{t}, u_{t+1}\right) \geq 0$ for $t=i, i+1$, the result follows immediately.

Definition 3.3. For a radio $k$-labeling $f$ of $P_{n}$ and a linear ordering $u_{0}, u_{1}, \ldots, u_{n-1}$ as in (3.1), two vertices $u_{i}$ and $u_{i+1}$ are called consecutive colored vertices under $f$ and their labels $f\left(u_{i}\right), f\left(u_{i+1}\right)$ are called consecutive radio $k$-coloring numbers. A radio labeling $f$ is said to be an alternating radio $k$-labeling if two consecutive colored vertices are in different branches.

Observation 3.4. From the above discussion, we may observe the following points under the Assumption 3.1:

(1) For an alternating labeling $f, \sigma(f)=J(f)$. Also if $f$ is not an alternating radio labeling, then $\sigma(f) \geq 2$ because in this case there exist at least one pair $u_{t}, u_{t+1}$ of vertices which are in same branch, i.e., $\phi\left(u_{t}, u_{t+1}\right) \geq 1$.

(2) If $n$ is even and the vertex $u_{i+1}$ is in highest level, then $J_{f}\left(u_{i}, u_{i+1}\right)+J_{f}\left(u_{i+1}, u_{i+2}\right) \geq$ 2 when $C \notin\left\{u_{i}, u_{i+2}\right\}$ and $i \neq n-2$ (by Lemma 3.2 using $L\left(u_{i+1}\right)=\frac{n}{2}$ ).

(3) If $n$ is odd and the vertex $u_{i+1}$ is in highest level, then $J_{f}\left(u_{i}, u_{i+1}\right)+J_{f}\left(u_{i+1}, u_{i+2}\right) \geq$ 1 when $C \notin\left\{u_{i}, u_{i+2}\right\}$ (by Lemma 3.2 using $L\left(u_{i+1}\right)=\frac{n-1}{2}$ ).

(4) For odd integer $n$, if $C \in\left\{u_{0}, u_{n-1}\right\}$ and $\left\{u_{0}, u_{n-1}\right\} \backslash C$ is not in highest level then $\sigma(f) \geq 1$ because in this case there exist at least one highest level vertex $u_{t}$ in the segment $u_{2}, u_{3}, \ldots, u_{n-2}$ such that $J_{f}\left(u_{t-1}, u_{t}\right)+J_{f}\left(u_{t}, u_{i+2}\right) \geq 1$.

(5) If $C \notin\left\{u_{0}, u_{n-1}\right\}$, then $L\left(u_{0}\right)+L\left(u_{n-1}\right) \geq 2$.

Theorem 3.5. Let $P_{n}$ be a path of odd number of vertices $n$ and let $f$ be any radio labeling of $P_{n}$ with first and last colored vertices are $u_{0}$ and $u_{m}$, respectively. Then

$$
\operatorname{span}_{f}\left(P_{n}\right) \geq \frac{(n-1)^{2}}{2}+f\left(u_{0}\right)+L\left(u_{0}\right)+L\left(u_{m}\right)+\sigma(f) .
$$

Proof. From Equation (3.3), the result follows immediately.

Corollary 3.6. Let $P_{n}$ be a path of odd number of vertices $n$ and let $f$ be any radio labeling of $P_{n}$ with first and last colored vertices are $u$ and $v$, respectively. If $u$ and $v$ are in same branch of $P_{n}$ and none of them are neither weight center nor highest level vertices, then

$$
\operatorname{span}_{f}\left(P_{n}\right) \geq \frac{(n-1)^{2}}{2}+f(u)+L(u)+L(v)+1 .
$$

Proof. Let the radio labeling $f$ induces the vertices of $P_{n}$ as $u=u_{0}, u_{1}, \ldots, u_{n-1}=v$. Without loss of generality, we take $u, v \in L\left(P_{n}\right)$. As $n$ is odd, $\left|L\left(P_{n}\right)\right|=\left|R\left(P_{n}\right)\right|$. Let $C$ be the weight center of $P_{n}$. Here $C \notin\left\{u_{0}, u_{n-1}\right\}$. Thus we consider $C=u_{r}$ for some $r \in\{1,2, \ldots, n-2\}$. Let $D_{1}=\left\{u_{0}, u_{1}, \ldots, u_{r-1}\right\}$ and $D_{2}=\left\{u_{r+1}, u_{r+2}, \ldots, u_{n-1}\right\}$. If 
one of $D_{1}$ or $D_{2}$ contains two consecutive colored vertices $u_{i}, u_{i+1}$ from same branch, then $\phi\left(u_{i}, u_{i+1}\right) \geqslant 1$. So $\sigma(f) \geqslant 2$. Now we consider the case when both $D_{1}$ and $D_{2}$ are alternating sequence of vertices. In this case as $u_{0}, u_{n-1} \in L\left(P_{n}\right)$ and $\left|L\left(P_{n}\right)\right|=\left|R\left(P_{n}\right)\right|$, so $u_{r-1}, u_{r+1} \in R\left(P_{n}\right)$. If none of $u_{0}$ or $u_{n-1}$ is the highest level $\frac{n-1}{2}$, then the $\frac{n-1}{2}$-level vertex in left branch, say, $u_{p} \in D_{1} \cup D_{2} \backslash\left\{u_{1}, u_{r-1}, u_{r+1}, u_{n-2}\right\}$. So applying Lemma 3.2, we have $J_{f}\left(u_{p-1}, u_{p}\right)+J_{f}\left(u_{p}, u_{p+1}\right) \geqslant 1$. Thus $\sigma(f) \geqslant 1$.

Theorem 3.7. Let $P_{n}$ be a path of even number of vertices $n$ and let $f$ be any radio labeling of $P_{n}$ with first and last colored vertices $u_{0}$ and $u_{m}$, respectively. Then

$$
\operatorname{span}_{f}\left(P_{n}\right) \geq \frac{(n-1)^{2}-1}{2}+f\left(u_{0}\right)+L\left(u_{0}\right)+L\left(u_{m}\right)+\sigma(f) .
$$

Proof. From Equation (3.3), the result follows immediately.

Remark 3.8. Liu and Zhu [25] have determined the exact value of radio number of path $P_{n}(n \geq 4)$ as $\frac{n^{2}}{2}-n+1$ if $n$ is even and $\frac{n^{2}+1}{2}-n+2$ if $n$ is odd. Thus the lower bound given in Theorems 3.5 and 3.7 coincide with the radio number of $P_{n}$.

Definition 3.9. Let $f: E \rightarrow F$ be a mapping from a set $E$ to a set $F$. For a set $A \subset E$, we call the mapping $\left.f\right|_{A}: A \rightarrow F$ as the restriction of $f$ on $A$.

Lemma 3.10. Let $f$ be any radio $k$-labeling of an $n$-vertex path $P_{n}$ with $n \geq k+1$. Then for any sub-path $P_{k+1}$ of $P_{n}, \operatorname{span}_{f}\left(P_{n}\right) \geq \operatorname{span}_{f}\left(P_{k+1}\right)$.

Proof. Let $f$ be a radio $k$-labeling of $P_{n}$. Since $P_{k+1}$ is a subpath of $P_{n}, V\left(P_{k+1}\right) \subset V\left(P_{n}\right)$. Let $g=\left.f\right|_{V\left(P_{k+1}\right)}$ be the restriction of $f$ on $V\left(P_{k+1}\right)$. Then $\operatorname{span}_{f}\left(P_{n}\right) \geqslant \operatorname{span}_{g}\left(P_{k+1}\right)$ and this is true for any radio $k$-labeling of $P_{n}$ and its restriction $g=\left.f\right|_{V\left(P_{k+1}\right)}$.

\section{Lower bound of $r c_{k}\left(P_{n}\right)$}

Theorem 4.1. Let $P_{n}$ be a path of order $n$. If $n \leq\left\lfloor\frac{3 k+1}{2}\right\rfloor$, then $r c_{k}\left(P_{n}\right) \geq\left\lfloor\frac{k^{2}}{2}\right\rfloor+n-k$.

Proof. The main key for the proof of this theorem is to search a sub-path $P_{k+1}^{*}$ of length $k$ whose radio number is at least $\left\lfloor\frac{k^{2}}{2}\right\rfloor+n-k$. Let $f$ be a radio- $k$-labeling of an $n$-vertex path $P_{n}: 0,1,2, \ldots, n-1$. As $1 \leq k \leq n-2$, it is always possible to find a sub-path $P_{k+1}$ of length $k$. Now we consider a sub-path $P_{k+1}^{0}: 0,1,2, \ldots, k$ of length $k$ as in Fig. 1. Rest of path $P_{n}$ is of length $\ell$, say. Then $n-1=k+\ell$. We also construct a sub-path $P_{k+1}^{\ell}: \ell, \ell+1, \ell+2, \ldots, \ell+k(=n-1)$ as in Fig. 1. If $k$ is even, then every sub-path of length $k$ has exactly one position of weight center. Otherwise there are two position of weight center. When $k$ is even, the weight centers $C_{0}$ and $C_{\ell}$ of sub-paths $P_{k+1}^{0}$ and $P_{k+1}^{\ell}$ are at the vertices $\frac{k}{2}$ and $\frac{k}{2}+\ell$ of path $P_{n}$, respectively (see Fig. 1 for an illustration). When $k$ is odd, position of $C_{0}$ is at the vertex $\frac{k-1}{2}$ or $\frac{k+1}{2}$ and position of $C_{\ell}$ is at the vertex $\frac{k-1}{2}+\ell$ or $\frac{k+1}{2}+\ell$ of path $P_{n}$ (see Fig. 3 for an illustration).

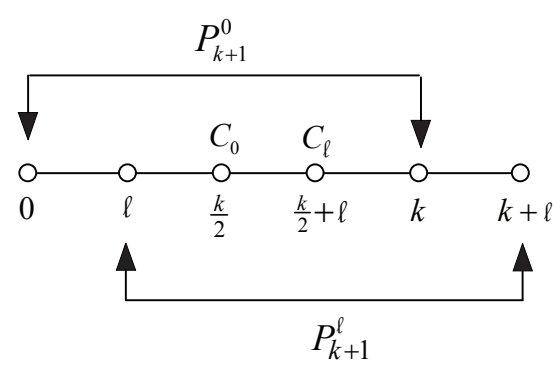

Figure 1. Sub-path construction of $P_{n}$ when $k$ is even. 
Since $n-1=k+\ell$ and $n \leq\left\lfloor\frac{3 k+1}{2}\right\rfloor$, thus $\ell \leq\left\lfloor\frac{k-1}{2}\right\rfloor$ and hence the weight center $C_{0}$ of sub-path $P_{k+1}^{0}$ belongs to the sub-path $P_{k+1}^{\ell}$. For radio- $k$-labeling $f$ of the path $P_{n}$, let $u$ be the initial colored vertex and $v$ be the maximum colored vertex. Then $f(u)=0$ and $\operatorname{span}_{f}\left(P_{n}\right)=f(v)$. Now we consider the positions of $u$ and $v$ on the path $P_{n}: 0,1,2, \ldots, n-1$.

Case I: $u$ or $v \in\{0,1,2, \ldots, \ell\}$. If $u \in\{0,1,2, \ldots, \ell\}$, then we can construct a sub-path $P_{k+1}^{u}: u, u+1, u+2, \ldots, u+k$ of length $k$ as in Fig. 2. As $u \leq \ell$, so $u+k \leq k+\ell=n-1$. Hence sub-path $P_{k+1}^{u}$ always exist in this case.

Subcase (a): $k$ is even. Here weight center, say, $C_{u}$ of $P_{k+1}^{u}$ is $u+\frac{k}{2}$. So $d\left(C_{u}, u\right)=$ $\frac{k}{2}=L(u)$ and $L(v)=d\left(C_{u}, v\right) \geq 0$. Applying Theorem 3.5 to path $P_{k+1}^{u}$, we have $\operatorname{span}_{f}\left(P_{k+1}^{u}\right) \geq \frac{k^{2}}{2}+\frac{k}{2} \geq \frac{k^{2}}{2}+n-k$ as $n \leq \frac{3 k}{2}=\left\lfloor\frac{3 k+1}{2}\right\rfloor$. If $v \in\{0,1,2, \ldots, \ell\}$, then for

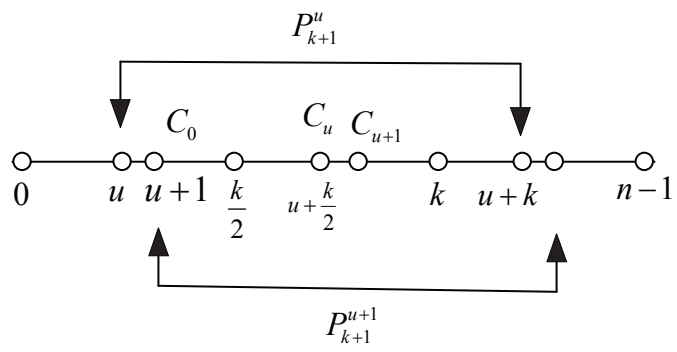

Figure 2. The sub-paths $P_{k+1}^{u}$ and $P_{k+1}^{u+1}$ of $P_{n}$.

the sub-path $P_{k+1}^{v}: v, v+1, v+2, \ldots, v+k$ of length $k$, by the same argument, one can easily prove that $\operatorname{span}_{f}\left(P_{k+1}^{v}\right) \geq \frac{k^{2}}{2}+\frac{k}{2} \geq \frac{k^{2}}{2}+n-k$.

Subcase (b): $k$ is odd. Here weight centers of $P_{k+1}^{u}$ are $u+\frac{k-1}{2}$ and $u+\frac{k+1}{2}$. Let us denote the weight center of $P_{k+1}^{u}$ by $C_{u}$. First we assume that $C_{u}=u+\frac{k-1}{2}$. If $C_{u}=v$ (the maximum colored vertex by $f$ ), then $d\left(C_{u}, u\right)+d\left(C_{u}, v\right)=\frac{k-1}{2}$. As the left branch $L\left(P_{k+1}^{u}\right)$ of $P_{k+1}^{u}$ has less number of vertices than the right branch $R\left(P_{k+1}^{u}\right)$ and first color vertex is in left branch, so $f$ can not be alternating radio labeling for the path $P_{k+1}^{u}$. Thus from Observation $3.4(1), \sigma(f) \geq 2$ and hence $\operatorname{span}_{f}\left(P_{k+1}^{u}\right) \geq \frac{k^{2}-1}{2}+\frac{k-1}{2}+2>\left\lfloor\frac{k^{2}}{2}\right\rfloor+n-k$. Otherwise, $C_{u} \neq v$. Then $d\left(C_{u}, v\right) \geq 1$ and from Theorem 3.7 we obtain, $\operatorname{span}_{f}\left(P_{k+1}^{u}\right) \geq$ $\frac{k^{2}-1}{2}+\frac{k+1}{2} \geq\left\lfloor\frac{k^{2}}{2}\right\rfloor+n-k$.

Next we assume that $C_{u}=u+\frac{k+1}{2}$. Then $d\left(C_{u}, u\right)+d\left(C_{u}, v\right) \geq \frac{k+1}{2}$. By applying Theorem 3.7 to the path $P_{k+1}^{u}$, we have $\operatorname{span}_{f}\left(P_{k+1}^{u}\right) \geq \frac{k^{2}-1}{2}+\frac{k+1}{2} \geq\left\lfloor\frac{k^{2}}{2}\right\rfloor+n-k$.

If $v \in\{0,1,2, \ldots, \ell\}$, then by the argument above for the sub-path $P_{k+1}^{v}: v, v+1, v+$ $2, \ldots, v+k$; we can easily prove that $\operatorname{span}_{f}\left(P_{k+1}^{v}\right) \geq \frac{k^{2}-1}{2}+\frac{k+1}{2} \geq\left\lfloor\frac{k^{2}}{2}\right\rfloor+n-k$.

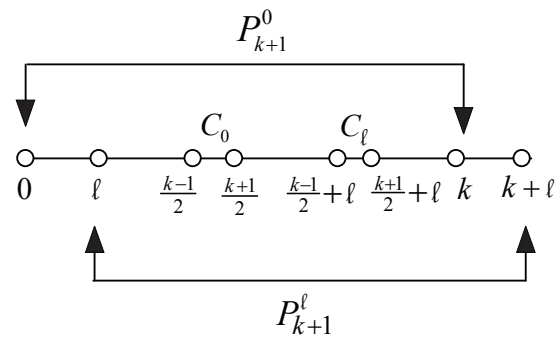

Figure 3. Sub-paths $P_{k+1}^{0}$ and $P_{k+1}^{\ell}$ of $P_{n}$. 
Case II: $u$ or $v \in\left\{\ell+1, \ell+2, \ldots,\left\lfloor\frac{k}{2}\right\rfloor\right\}$. If $u \in\left\{\ell+1, \ell+2, \ldots,\left\lfloor\frac{k}{2}\right\rfloor\right\}$, then we can construct a sub-path $P_{k+1}^{\ell}: \ell, \ell+1, \ell+2, \ldots, \ell+k$ of length $k$.

Subcase (a): $k$ is even. Here weight center $C_{\ell}$ of path $P_{k+1}^{\ell}$ is $\ell+\frac{k}{2}$. So $d\left(C_{\ell}, u\right)=$ $\frac{k}{2}+\ell-u=L(u)$. Thus applying Theorem 3.5 to path $P_{k+1}^{\ell}$, we have $\operatorname{span}_{f}\left(P_{k+1}^{\ell}\right) \geq \frac{k^{2}}{2}+\frac{k}{2}+$ $\ell-u+L(v)+\sigma(f)$. Since $\ell=n-k-1$ and $u \leq \frac{k}{2}, \operatorname{span}_{f}\left(P_{k+1}^{\ell}\right) \geq \frac{k^{2}}{2}+n-k-1+L(v)+\sigma(f)$. If $L(v)=d\left(C_{\ell}, v\right) \geq 1$, then $\operatorname{span}_{f}\left(P_{k+1}^{\ell}\right) \geq \frac{k^{2}}{2}+n-k+\sigma(f) \geq\left\lfloor\frac{k^{2}}{2}\right\rfloor+n-k$. Otherwise, $d\left(C_{\ell}, v\right)=0$. Thus we have $v=\frac{k}{2}+\ell$. Therefore the maximum colored vertex $v$ is the weight center of path $P_{k+1}^{\ell}$ and the minimum colored vertex $u$ is not the highest level. Thus from Observation $3.4(4)$, we have $\sigma(f) \geq 1$ for sub-path $P_{k+1}^{\ell}$. Therefore for the sub-path $P_{k+1}^{\ell}$, we have $\operatorname{span}_{f}\left(P_{k+1}^{\ell}\right) \geq\left\lfloor\frac{k^{2}}{2}\right\rfloor+n-k$.

If $v \in\left\{\ell+1, \ell+2, \ldots, \frac{k}{2}\right\}$, then for the sub-path $P_{k+1}^{\ell}$, by the same argument, one can easily prove that $\operatorname{span}_{f}\left(P_{k+1}^{\ell}\right) \geq\left\lfloor\frac{k^{2}}{2}\right\rfloor+n-k$.

Subcase (b): $k$ is odd. In this subcase weight center $C_{\ell}$ of $P_{k+1}^{\ell}$ is $\ell+\frac{k-1}{2}$ or $\ell+\frac{k+1}{2}$. First we assume that weight center $C_{\ell}=\ell+\frac{k-1}{2}$. Then the left branch $L\left(P_{k+1}^{\ell}\right)$ has less number of vertices than the right branch $R\left(P_{k+1}^{\ell}\right)$ of $P_{k+1}^{\ell}$. Now, $d\left(C_{\ell}, u\right)+d\left(C_{\ell}, v\right)=$ $\frac{k-1}{2}+\ell-u+d\left(C_{\ell}, v\right)$. By Theorem 3.7, we have $\operatorname{span}_{f}\left(P_{k+1}^{\ell}\right) \geq \frac{k^{2}-1}{2}+\frac{k-1}{2}+\ell-u+d\left(C_{\ell}, v\right)$. If $d\left(C_{\ell}, v\right) \geq 1$, then we get $\operatorname{span}_{f}\left(P_{k+1}^{\ell}\right) \geq \frac{k^{2}-1}{2}+\ell+1=\left\lfloor\frac{k^{2}}{2}\right\rfloor+n-k$ as $u \leq \frac{k-1}{2}$. Otherwise, $d\left(C_{\ell}, v\right)=0$, i.e., $C_{\ell}=v$. Then $d\left(C_{\ell}, u\right)+d\left(C_{\ell}, v\right)=\frac{k-1}{2}+\ell-u$. Since the first colored vertex $u$ is in the left branch $L\left(P_{k+1}^{\ell}\right)$ of $P_{k+1}^{\ell}$ and the maximum colored vertex $v$ is the centroid, so $f$ can not be an alternating radio labeling of $P_{k+1}^{\ell}$ due to the same fact as described in Case $I$. By applying Theorem 3.7 to $P_{k+1}^{\ell}$ with $\sigma(f) \geq 2$, we

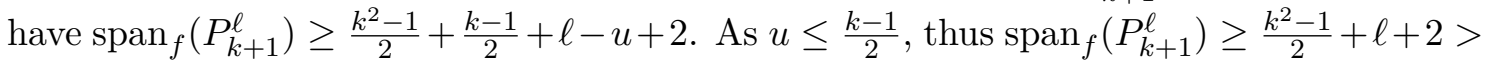
$\left\lfloor\frac{k^{2}}{2}\right\rfloor+n-k$.

Next we assume that weight center $C_{\ell}$ of $P_{k+1}^{\ell}$ is $\ell+\frac{k+1}{2}$. Then $d\left(C_{\ell}, u\right)+d\left(C_{\ell}, v\right) \geq$ $\frac{k+1}{2}+\ell-u$. As $u \leq \frac{k-1}{2}$, hence $\operatorname{span}_{f}\left(P_{k+1}^{\ell}\right) \geq \frac{k^{2}-1}{2}+\ell+1=\left\lfloor\frac{k^{2}}{2}\right\rfloor+n-k$.

If $v \in\left\{\ell+1, \ell+2, \ldots, \frac{k-1}{2}\right\}$, then for the sub-path $P_{k+1}^{\ell}$, by the same argument we can easily prove that $\operatorname{span}_{f}\left(P_{k+1}^{\ell}\right) \geq\left\lfloor\frac{k^{2}}{2}\right\rfloor+n-k$.

Case III: Both $u$ and $v$ lie in $\left\{\left\lfloor\frac{k}{2}\right\rfloor+1,\left\lfloor\frac{k}{2}\right\rfloor+2, \ldots,\left\lfloor\frac{k}{2}\right\rfloor+\ell\right\}$. In this case both $u$ and $v$ are in $P_{k+1}^{0}$ as well as $P_{k+1}^{\ell}$.

Subcase (a): $k$ is even. We have $L(u)=d\left(C_{0}, u\right)=u-\frac{k}{2}$ and $L(v)=d\left(C_{0}, v\right)=v-\frac{k}{2}$. As both first and last colored vertices are in same side (right side) of $P_{k+1}^{0}$ and none of them are neither the weight center nor the highest level vertices, so by Corollary 3.6, we have

$$
\operatorname{span}_{f}\left(P_{k+1}^{0}\right) \geq \frac{k^{2}}{2}+u+v-k+1
$$

Again for the sub-path $P_{k+1}^{\ell}$, the weight center $C_{\ell}$ is the vertex $\frac{k}{2}+\ell$. So $L(u)=$ $d\left(C_{\ell}, u\right)=\frac{k}{2}+\ell-u$ and $L(v)=d\left(C_{\ell}, v\right)=\frac{k}{2}+\ell-v$. First we assume that $v \neq \frac{k}{2}+\ell$. Since both first and last colored vertices are in the same side (left side) of path $P_{k+1}^{\ell}$ and none of them are neither the weight center nor the highest level vertices, so by Corollary 
3.6, we have

$$
\operatorname{span}_{f}\left(P_{k+1}^{\ell}\right) \geq \frac{k^{2}}{2}+k+2 \ell-(u+v)+1 .
$$

Next we assume that $v=\frac{k}{2}+\ell$ of path $P_{k+1}^{\ell}$. Then by the similar argument as in Case II, we can show that $\sigma(f) \geq 1$. By applying Theorem 3.5 to path $P_{k+1}^{\ell}$, we have

$$
\operatorname{span}_{f}\left(P_{k+1}^{\ell}\right) \geq \frac{k^{2}}{2}+1+k+2 \ell-(u+v) .
$$

By simple calculations one can easily prove that $\max \{u+v-k, k+2 \ell-(u+v)\} \geq \ell=$ $n-k-1$. Thus we have

$$
\begin{aligned}
\operatorname{span}_{f}\left(P_{n}\right) & \geq \max \left\{\operatorname{span}_{f}\left(P_{k+1}^{0}\right), \operatorname{span}_{f}\left(P_{k+1}^{\ell}\right)\right\} \\
& \geq \frac{k^{2}}{2}+1+\max \{u+v-k, k+2 \ell-(u+v)\} \\
& \geq\left\lfloor\frac{k^{2}}{2}\right\rfloor+n-k .
\end{aligned}
$$

Subcase (b): $k$ is odd. If the weight center $C_{0}$ is $\frac{k-1}{2}$ of the path $P_{k+1}^{0}$, then $d\left(C_{0}, u\right)+$ $d\left(C_{0}, v\right)=u+v-k+1$. Then by Theorem 3.7, we have

$$
\operatorname{span}_{f}\left(P_{k+1}^{0}\right) \geq \frac{k^{2}-1}{2}+u+v-k+1
$$

Otherwise, the weight center $C_{0}$ is $\frac{k+1}{2}$. Then the right branch $R\left(P_{k+1}^{0}\right)$ has less number of vertices than the left branch $L\left(P_{k+1}^{0}\right)$ of $P_{k+1}^{0}$ and $d\left(C_{0}, u\right)+d\left(C_{0}, v\right)=u+v-k-1$. As both $u$ and $v$ are in $R\left(P_{k+1}^{0}\right)$, so $\sigma_{f}\left(P_{k+1}^{0}\right) \geq 2$ (since $f$ can not be alternating radio labeling for the path $P_{k+1}^{0}$ as described in Case II of this theorem). By Theorem 3.7, we have

$$
\operatorname{span}_{f}\left(P_{k+1}^{0}\right) \geq \frac{k^{2}-1}{2}+u+v-k+1
$$

By the similar argument to the path $P_{k+1}^{\ell}$ with the weight centers $\frac{k-1}{2}+\ell$ and $\frac{k+1}{2}+\ell$, we obtain

$$
\operatorname{span}_{f}\left(P_{k+1}^{\ell}\right) \geq \frac{k^{2}-1}{2}+k+1+2 \ell-u-v .
$$

It is easy to prove that $\max \{u+v-k+1, k-1+2 \ell-(u+v)\} \geq \ell+1=n-k$. Thus we have

$$
\begin{aligned}
\operatorname{span}_{f}\left(P_{n}\right) & \geq \max \left\{\operatorname{span}_{f}\left(P_{k+1}^{0}\right), \operatorname{span}_{f}\left(P_{k+1}^{\ell}\right)\right\} \\
& \geq \frac{k^{2}-1}{2}+1+\max \{u+v-k, k+2 \ell-(u+v)\} \\
& \geq\left\lfloor\frac{k^{2}}{2}\right\rfloor+n-k .
\end{aligned}
$$


Case IV: $u$ or $v \in\left\{\left\lfloor\frac{k}{2}\right\rfloor+\ell+1,\left\lfloor\frac{k}{2}\right\rfloor+\ell+2, \ldots, k\right\}$. This case is similar to Case II. For the sub-path $P_{k+1}^{0}$, by the same argument as used in Case II, one can easily prove that $\operatorname{span}_{f}\left(P_{k+1}^{0}\right) \geq\left\lfloor\frac{k^{2}}{2}\right\rfloor+n-k$.

Case V: $u$ or $v \in\{k+1, k+2, \ldots, n-1\}$. For the sub-path $P_{k+1}^{u-k}: u-k, u-k+1, u-$ $k+2, \ldots, u$; by the same argument as used in Case I, we have $\operatorname{span}_{f}\left(P_{k+1}^{u-k}\right) \geq\left\lfloor\frac{k^{2}}{2}\right\rfloor+n-k$.

Finally we conclude that for any radio- $k$-labeling $f$ of path $P_{n}, \operatorname{span}_{f}\left(P_{n}\right) \geq\left\lfloor\frac{k^{2}}{2}\right\rfloor+n-k$ and hence $r c_{k}\left(P_{n}\right) \geq\left\lfloor\frac{k^{2}}{2}\right\rfloor+n-k$.

Corollary 4.2. For an $n$-vertex path $P_{n}$ with even integer $k$,

$$
r c_{k}\left(P_{n}\right) \geq \frac{k^{2}}{2}+\min \left\{n-k, \frac{k}{2}\right\} .
$$

Proof. The value of $\min \left\{n-k, \frac{k}{2}\right\}$ is $n-k$ or $\frac{k}{2}$ according as $n \leq \frac{3 k}{2}$ and $n \geq \frac{3 k}{2}$. Since $r c_{k}\left(P_{n}\right) \geq r c_{k}\left(P_{m}\right)$ for $n \geq m$, the result is follows from Theorem 4.1.

Corollary 4.3. For an $n$-vertex path $P_{n}$ and odd integer $k, r c_{k}\left(P_{n}\right) \geq \frac{k^{2}-1}{2}+\min \left\{n-k, \frac{k+1}{2}\right\}$.

Proof. The value of $\min \left\{n-k, \frac{k+1}{2}\right\}$ is $n-k$ or $\frac{k+1}{2}$ according as $n \leq \frac{3 k+1}{2}$ and $n \geq \frac{3 k+1}{2}$. Since $r c_{k}\left(P_{n}\right) \geq r c_{k}\left(P_{m}\right)$ for $n \geq m$, the result follows from Theorem 4.1.

Theorem 4.4. Let $P_{n}$ be a path of order $n$ with even integer $k$. If $n \geq \frac{3 k}{2}+2$, then $r c_{k}\left(P_{n}\right) \geq \frac{k^{2}}{2}+\frac{k}{2}+1$.

Proof. We have $r c_{k}\left(P_{n}\right) \geq r c_{k}\left(P_{m}\right)$ for $n \geq m$. Thus we prove this theorem for $n=\frac{3 k}{2}+2$. Let $f$ be an optimal radio $k$-labeling of path $P_{n}$, where $n=\frac{3 k}{2}+2$. Also let the minimum color and the maximum color (say, $m$ and $M$, respectively) assigned by $f$ for the path $P_{n}: 0,1,2, \ldots, n-1$ are attained at vertices $u$ and $v$ of the path $P_{n}$, respectively. Now we consider the following cases depending on the positions of $u$ and $v$ in the path $P_{n}$.

Case I: $u$ or $v \in\left\{0,1,2, \ldots, \frac{k}{2}\right\}$. If $u \in\left\{0,1, \ldots, \frac{k}{2}\right\}$, then we can construct a sub-path $P_{k+1}^{u}: u, u+1, u+2, \ldots, u+k$ of length $k$ as in Fig. 2. Since $u \leq \frac{k}{2}$, we have $u+k \leq n-2$. Hence the sub-path $P_{k+1}^{u}$ always exist in this case. Let $C_{u}\left(=u+\frac{k}{2}\right)$ be the weight center of path $P_{k+1}^{u}$. So $d\left(C_{u}, u\right)=\frac{k}{2}$ and $d\left(C_{u}, v\right) \geq 0$. If $d\left(C_{u}, v\right) \geq 1$, then applying Theorem 3.5 to path $P_{k+1}^{u}$, we have $\operatorname{span}_{f}\left(P_{k+1}^{u}\right) \geq \frac{k^{2}}{2}+\frac{k}{2}+1$ and hence the result follows. Otherwise, $d\left(C_{u}, v\right)=0$. Therefore the maximum color $M$ attains at $C_{u}$, weight center of path $P_{k+1}^{u}$. Now we construct an another sub-path $P_{k+1}^{u+1}: u+1, u+2, u+3, \ldots, u+k+1$ of length $k$ with starting vertex $u+1$ of path $P_{n}$ (see Fig. 2). For this sub-path the weight center, denoted by $C_{u+1}$, is the vertex $u+\frac{k}{2}+1$ and the maximum color attains at the vertex $u+\frac{k}{2}=v$. Since the minimum colored vertex $u$ of path $P_{n}$ is not in the sub-path $P_{k+1}^{u+1}$, so let $m^{\prime}$ be the minimum color assigned by $f$ for the sub-path $P_{k+1}^{u+1}$ and $m^{\prime}$ attains at the vertex $u^{\prime} \in V\left(P_{k+1}^{u+1}\right)$, say. Here obviously $m^{\prime} \geq m$. We now consider the following two subcases:

Subcase (a): $u^{\prime}$ is in right half of $C_{u+1}$. Let $u^{\prime}=u+\frac{k}{2}+1+t$ with $0 \leq t \leq \frac{k}{2}$. Then $d\left(u, u^{\prime}\right)=t+\frac{k}{2}+1$ and $d\left(C_{u+1}, u^{\prime}\right)=t$. The color difference of the vertices $u$ and $u^{\prime}$ is 
$m^{\prime}-m$. From the radio $k$-labeling condition $m^{\prime}-m \geq \frac{k}{2}-t$. Thus applying Theorem 3.5 to path $P_{k+1}^{u+1}$, we have $\operatorname{span}_{f}\left(P_{k+1}^{u+1}\right) \geq \frac{k^{2}}{2}+d\left(C_{u+1}, u^{\prime}\right)+d\left(C_{u+1}, v\right)+m^{\prime} \geq \frac{k^{2}}{2}+\frac{k}{2}+1$.

Subcase (b): $u^{\prime}$ is in left half of $C_{u+1}$. Let $u^{\prime}=u+\frac{k}{2}+1-t$ with $2 \leq t \leq \frac{k}{2}$. Then $d\left(u, u^{\prime}\right)=\frac{k}{2}+1-t$ and $d\left(C_{u+1}, u^{\prime}\right)=t$. The color difference between $u$ and $u^{\prime}$ is $m^{\prime}-m$. From the radio $k$-labeling condition $m^{\prime}-m \geq \frac{k}{2}+t$. Thus applying Theorem 3.5 to path $P_{k+1}^{u+1}$, we have $\operatorname{span}_{f}\left(P_{k+1}^{u+1}\right) \geq \frac{k^{2}}{2}+d\left(C_{u+1}, u^{\prime}\right)+d\left(C_{u+1}, v\right)+m^{\prime} \geq \frac{k^{2}}{2}+\frac{k}{2}+2 t+1$.

Thus if $u \in\left\{0,1,2, \ldots, \frac{k}{2}\right\}$, there always exist a sub-path $P$ of length $k$ with $\operatorname{span}_{f}(P) \geq$ $\frac{k^{2}}{2}+\frac{k}{2}+1$.

If $v \in\left\{0,1,2, \ldots, \frac{k}{2}\right\}$, then for the sub-path $P_{k+1}^{v}: v, v+1, v+2, \ldots, v+k$, by the same argument, one can easily prove the required result.

Case II: Both $u, v \in\left\{\frac{k}{2}+1, \frac{k}{2}+2, \ldots, k\right\}$. Construct two sub-paths $P_{k+1}^{0}: 0,1, \ldots, k$ and $P_{k+1}^{\frac{k}{2}+1}: \frac{k}{2}+1, \frac{k}{2}+2, \ldots, \frac{3 k}{2}+1$. Here the weight centers of $P_{k+1}^{0}$ and $P_{k+1}^{\frac{k}{2}+1}$ are at

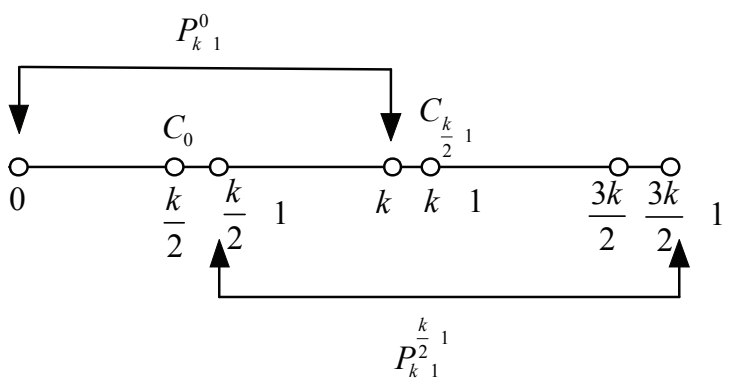

Figure 4. The sub-paths $P_{k+1}^{0}$ and $P_{k+1}^{\frac{k}{2}+1}$.

the vertices $\frac{k}{2}$ and $k+1$ of $P_{n}$, respectively. Let us denote these weight centers by $C_{0}$ and $C_{\frac{k}{2}+1}$, respectively. In this case both $u$ and $v$ are in $P_{k+1}^{0}$ as well as $P_{k+1}^{\frac{k}{2}+1}$. Here $d\left(C_{0}, u\right)=d\left(\frac{k}{2}, u\right)=u-\frac{k}{2} ; d\left(C_{0}, v\right)=d\left(\frac{k}{2}, v\right)=v-\frac{k}{2} ; d\left(C_{\frac{k}{2}+1}, u\right)=d(k+1, u)=k+1-u$ and $d\left(C_{\frac{k}{2}+1}, v\right)=d(k+1, v)=k+1-v$. Then applying Theorem 3.5 to the paths $P_{k+1}^{0}$ and $P_{k+1}^{\frac{k}{2}+1}$ each of length $k$, we have

$$
\operatorname{span}_{f}\left(P_{k+1}^{0}\right) \geq \frac{k^{2}}{2}+u+v-k \text { and } \operatorname{span}_{f}\left(P_{k+1}^{\frac{k}{2}+1}\right) \geq \frac{k^{2}}{2}+2(k+1)-u-v .
$$

By simple calculations, one can easily prove that $\max \{u+v-k, 2(k+1)-u-v\} \geq \frac{k}{2}+1$. Since $\operatorname{span}_{f}\left(P_{n}\right) \geq \max \left\{\operatorname{span}_{f}\left(P_{k+1}^{0}\right), \operatorname{span}_{f}\left(P_{k+1}^{\frac{k}{2}+1}\right)\right\}$, therefore

$$
\operatorname{span}_{f}\left(P_{n}\right) \geq \frac{k^{2}}{2}+\max \{u+v-k, 2(k+1)-u-v\} \geq \frac{k^{2}}{2}+\frac{k}{2}+1 .
$$

Case III: $u$ or $v \in\left\{k+1, k+2, \ldots, \frac{3 k}{2}+1\right\}$. This case is similar to Case I if we reverse the vertex labeling of path $P_{n}$ by the operation $j=\frac{3 k}{2}+1-i, 0 \leq i \leq \frac{3 k}{2}+1$. This completes the proof of the theorem.

Theorem 4.5. Let $P_{n}$ be a path of order $n$ with odd integer $k$. If $n \geq \frac{5 k+1}{2}$, then $\operatorname{rc}_{k}\left(P_{n}\right) \geq$ $\frac{k^{2}+k}{2}+1$. 
Proof. This theorem can be prove by similar argument as given in Theorem 4.4.

Remark 4.6. The existing lower bound of $r c_{k}\left(P_{n}\right)$ is $\frac{k^{2}+4}{2}$ for even integer $k$ and $\frac{k^{2}+1}{2}$ for odd integer $k$ (see, [14]). But the results presented in Section 4 gives an improved lower bound of $r c_{k}\left(P_{n}\right)$ for even integer $k$ as $\frac{k^{2}}{2}+\min \left\{n-k, \frac{k}{2}\right\}$ or $\frac{k^{2}}{2}+\frac{k}{2}+1$ according as $n \leq \frac{3 k}{2}$ and $n \geq \frac{3 k}{2}+2$. For odd integer $k$, improved lower bound presented here as $\frac{k^{2}-1}{2}+\min \left\{n-k, \frac{k+1}{2}\right\}$ or $\frac{k^{2}+k}{2}+1$ according as $n \leq \frac{5 k-1}{2}$ and $n \geq \frac{5 k+1}{2}$.

\section{Radio $k$-chromatic number of $P_{n}$ when $k$ is even and $n \leq \frac{5 k}{2}+2$}

In this section, we give the exact value of $r c_{k}\left(P_{n}\right)$ when $k$ is even and $n \leq \frac{5 k}{2}+2$.

Theorem 5.1. For an $n$-vertex path $P_{n}$ and an even integer $k$,

$$
r c_{k}\left(P_{n}\right)= \begin{cases}\frac{k^{2}}{2}+n-k & \text { if } n \leq \frac{3 k}{2}, \\ \frac{k^{2}}{2}+\frac{k}{2} & \text { if } n=\frac{3 k}{2}+1, \\ \frac{k^{2}}{2}+\frac{k}{2}+1 & \text { if } \frac{3 k}{2}+2 \leq n \leq \frac{5 k}{2}+2 .\end{cases}
$$

Proof. Let $V\left(P_{n}\right)=\{0,1,2, \ldots, n-1\}$ be the vertex set of an $n$-vertex path $P_{n}$. By Theorems 4.1 and 4.4 with Corollary 4.2 , we have

$$
r c_{k}\left(P_{n}\right) \geq \begin{cases}\frac{k^{2}}{2}+n-k & \text { if } n \leq \frac{3 k}{2} \\ \frac{k^{2}}{2}+\frac{k}{2} & \text { if } n=\frac{3 k}{2}+1, \\ \frac{k^{2}}{2}+\frac{k}{2}+1 & \text { if } \frac{3 k}{2}+2 \leq n \leq \frac{5 k}{2}+2 .\end{cases}
$$

To prove the equality we have to give an optimal $k$-labeling with this required span. To define optimal $k$-labelings we consider the following three cases depending on the values of $n$ as stated in this theorem.

Case I: $n \leq \frac{3 k}{2}$. Let $k=2 p$ and $\ell$ be a positive integer such that $k+\ell=n-1$ with $0<\ell<p$. Define a mapping $f: V\left(P_{n}\right) \rightarrow\{0,1,2, \ldots\}$ as follows:

$$
\begin{aligned}
f(i) & =p+1+i(2 p+1), 0 \leq i \leq \ell-2 ; \\
f(\ell-1) & =2 p^{2}-p+\ell+1 ; \\
f(\ell+j) & =p+2+(\ell+j-1)(2 p+1), 0 \leq j \leq p-\ell-1 ; \\
f(p+m) & =m(2 p+1), 0 \leq m \leq \ell-1 ; \\
f(p+\ell) & =2 p^{2}+\ell+1 ; \\
f(p+\ell+1+t) & =(\ell+t)(2 p+1)+1,0 \leq t \leq p-\ell-1 ; \\
f(2 p+1+r) & =p+r(2 p+1), 0 \leq r \leq \ell-1 .
\end{aligned}
$$

One can easily show that $f$ satisfies the radio $k$-labeling condition. Thus we have $\operatorname{span}_{f}\left(P_{n}\right)=$ $2 p^{2}+\ell+1=\frac{k^{2}}{2}+n-k$.

Case II: $n=\frac{3 k}{2}+1$. In this case we define a mapping $f: V\left(P_{n}\right) \rightarrow\{0,1,2, \ldots\}$ as follows: 


$$
\begin{aligned}
f(i) & =\frac{k}{2}+1+i(k+1), 0 \leq i \leq \frac{k}{2}-1 ; \\
f\left(\frac{k}{2}+j\right) & =j(k+1), 0 \leq j \leq \frac{k}{2} \\
f(k+\ell+1) & =\frac{k}{2}+\ell(k+1), 0 \leq \ell \leq \frac{k}{2}-1 .
\end{aligned}
$$

It is easy to show that $f$ satisfies the radio- $k$-labeling condition. Here clearly $\operatorname{span}_{f}\left(P_{n}\right)=$ $\frac{k^{2}}{2}+\frac{k}{2}$.

Case III: $\frac{3 k}{2}+2 \leq n \leq \frac{5 k}{2}+2$. Define a mapping $f: V\left(P_{n}\right) \rightarrow\{0,1,2, \ldots\}$ as follows:

$$
\begin{aligned}
f(i) & =\frac{k}{2}+2+i(k+1), 0 \leq i \leq \frac{k}{2}-1 ; \\
f\left(\frac{k}{2}+j\right) & =j(k+1)+1, \quad 0 \leq j \leq \frac{k}{2} \\
f(k+\ell+1) & =f(\ell)-1, \quad 0 \leq \ell \leq \frac{k}{2}-1 ; \\
f\left(\frac{3 k}{2}+m\right) & =f\left(\frac{k}{2}+m-1\right)-1,1 \leq m \leq n-1-\frac{3 k}{2} .
\end{aligned}
$$

It is easy to show that $f$ satisfies the radio- $k$-labeling condition. Thus we have $\operatorname{span}_{f}\left(P_{n}\right)=$ $\frac{k^{2}}{2}+\frac{k}{2}+1$. This completes the proof of the theorem.

Example 5.2. An optimal radio 8-labeling of $P_{20}$ and radio 14-labeling of $P_{19}$ have given in Fig. 5 and Fig. 6, respectively.

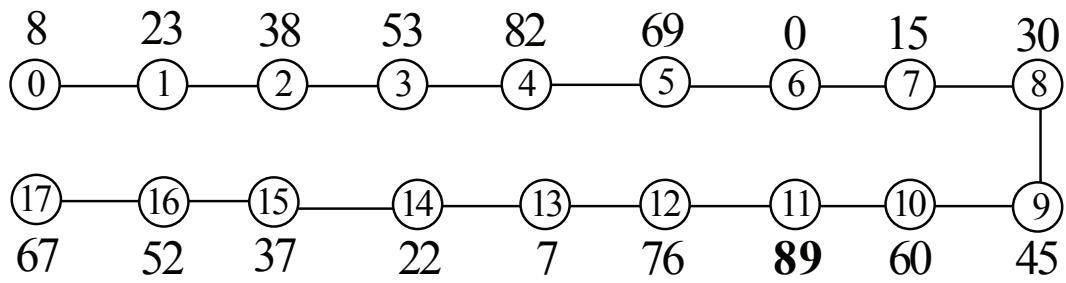

Figure 5. A radio 8-labeling of $P_{20}$ with span 37 .

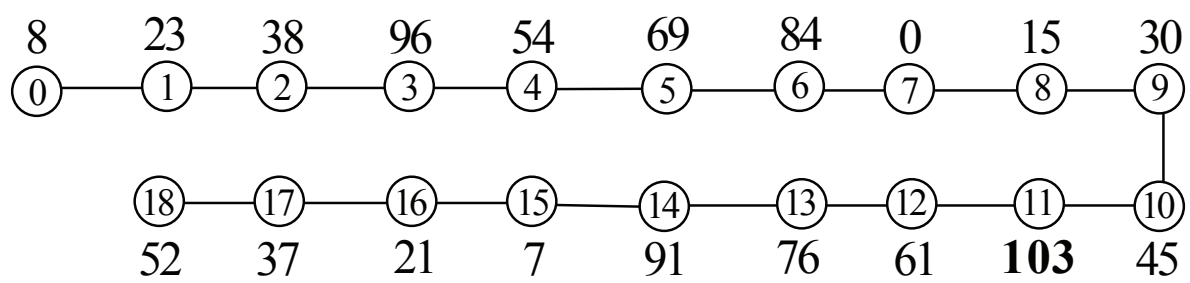

Figure 6. A radio 14-labeling of $P_{19}$ with span 103. 
6. Radio $k$-chromatic number of $P_{n}$ when $k$ is odd and $k+2 \leq n \leq \frac{7 k-1}{2}$

In this section, we give the exact value of $r c_{k}\left(P_{n}\right)$ when $k$ is odd and $k+2 \leq n \leq \frac{7 k-1}{2}$.

Theorem 6.1. For an $n$-vertex path $P_{n}$ and an odd integer $k$,

$$
r c_{k}\left(P_{n}\right)= \begin{cases}\frac{k^{2}-1}{2}+n-k & \text { if } k+2 \leq n \leq \frac{3 k-1}{2}, \\ \frac{k^{2}+k}{2} & \text { if } \frac{3 k+1}{2} \leq n \leq \frac{5 k-1}{2} \\ \frac{k^{2}+k}{2}+1 & \text { if } \frac{5 k+1}{2} \leq n \leq \frac{7 k-1}{2}\end{cases}
$$

Proof. From Corollary 4.3 and Theorem 4.5, we have the following:

$$
r c_{k}\left(P_{n}\right) \geq \begin{cases}\frac{k^{2}-1}{2}+n-k & \text { if } k+2 \leq n \leq \frac{3 k-1}{2} \\ \frac{k^{2}+k}{2} & \text { if } \frac{3 k+1}{2} \leq n \leq \frac{5 k-1}{2} \\ \frac{k^{2}+k}{2}+1 & \text { if } n \geq \frac{5 k+1}{2}\end{cases}
$$

To prove equality in (6.1), we have to define a radio $k$-labeling with the span as specified in this theorem. For the cases $\frac{3 k+1}{2} \leq n \leq \frac{5 k-1}{2}$ and $\frac{5 k+1}{2} \leq n \leq \frac{7 k-1}{2}$, it is sufficient to show that there exist radio $k$-labelings $f$ and $g$ for the paths $P_{\frac{5 k-1}{2}}$ and $P_{\frac{7 k-1}{2}}$ with spans $\frac{k^{2}+k}{2}$ and $\frac{k^{2}+k}{2}+1$, respectively (because these are the lower bounds and $r c_{k}\left(P_{n}\right) \geq r c_{k}\left(P_{m}\right)$ for $n \geq m)$.

Let the vertex set of an $n$-vertex path $P_{n}$ be $V\left(P_{n}\right)=\{0,1, \ldots, n-1\}$. Also let $k=2 p+1$ and $\ell$ be a positive integer such that $k+\ell=n-1$. We consider the following three cases:

Case I: $k+2 \leq n \leq \frac{3 k-1}{2}$. Since $n \leq \frac{3 k-1}{2}$, we have $\ell<p$. Define a mapping $f: V\left(P_{n}\right) \rightarrow$ $\{0,1,2, \ldots\}$ as follows:

$$
\begin{aligned}
f(i) & =p+2+i(2 p+3), 0 \leq i \leq \ell-1 ; \\
f(\ell) & =2 p^{2}+p+\ell ; \\
f(\ell+1+j) & =p+3+(\ell+j)(2 p+3), 0 \leq j \leq p-\ell-2 ; \\
f(p+m) & =m(2 p+3), 0 \leq m \leq \ell ; \\
f(p+\ell+1) & =2 p^{2}+2 p+\ell+1 ; \\
f(p+\ell+2+t) & =(\ell+t+1)(2 p+3)+1,0 \leq t \leq p-\ell-2 ; \\
f(2 p+1+r) & =p+1+r(2 p+3), 0 \leq r \leq \ell .
\end{aligned}
$$


One can easily check that $f$ satisfies the radio $k$-labeling condition. Thus we have $\operatorname{span}_{f}\left(P_{n}\right)=2 p^{2}+2 p+\ell+1=\frac{k^{2}-1}{2}+n-k$ as $k=2 p+1$.

Case II: $\frac{3 k+1}{2} \leq n \leq \frac{5 k-1}{2}$. As discuss above it is sufficient to define a radio $k$-labeling of $P_{n}$ only for $n=\frac{5 k-1}{2}$. We construct a radio $k$-labeling $f$ of $P_{\frac{5 k-1}{2}}$ as follows:

$$
\begin{aligned}
f(i) & =\frac{k+5}{2}+i(k+2), 0 \leq i \leq \frac{k-3}{2} ; \\
f\left(\frac{k-1}{2}+j\right) & =j(k+2)+1,0 \leq j \leq \frac{k-1}{2} ; \\
f(k+\ell) & =\frac{k+3}{2}+\ell(k+2), 0 \leq \ell \leq \frac{k-3}{2} ; \\
f\left(\frac{3 k-1}{2}+m\right) & =m(k+2), 0 \leq m \leq \frac{k-1}{2} ; \\
f(2 k+p) & =\frac{k+1}{2}+p(k+2), 0 \leq p \leq \frac{k-3}{2} .
\end{aligned}
$$

It is easy to see that $f$ satisfy the radio- $k$-labeling condition. Here clearly $\operatorname{span}_{f}\left(P_{n}\right)=$ $f(k-1)=\frac{k^{2}+k}{2}$. It is also noted that this radio $k$-labeling scheme will work for any path $P_{n}$ with $\frac{3 k+1}{2} \leq n \leq \frac{5 k-1}{2}$.

Case III: $\frac{5 k+1}{2} \leq n \leq \frac{7 k-1}{2}$. As discuss above it is sufficient to define a radio $k$-labeling of $P_{n}$ only for $n=\frac{7 k-1}{2}$. We construct a radio $k$-labeling $f$ of $P_{\frac{7 k-1}{2}}$ as follows:

$$
\begin{aligned}
f(i) & =\frac{k+7}{2}+i(k+2), 0 \leq i \leq \frac{k-3}{2} ; \\
f\left(\frac{k-1}{2}+j\right) & =j(k+2)+2,0 \leq j \leq \frac{k-1}{2} ; \\
f(k+\ell) & =\frac{k+5}{2}+\ell(k+2), 0 \leq \ell \leq \frac{k-3}{2} ; \\
f\left(\frac{3 k-1}{2}+m\right) & =m(k+2)+1,0 \leq m \leq \frac{k-1}{2} ; \\
f(2 k+p) & =\frac{k+3}{2}+p(k+2), 0 \leq p \leq \frac{k-3}{2} ; \\
f\left(\frac{5 k-1}{2}+q\right) & =q(k+2), 0 \leq q \leq \frac{k-1}{2} ; \\
f(3 k+r) & =\frac{k+1}{2}+r(k+2), 0 \leq r \leq \frac{k-3}{2} .
\end{aligned}
$$

Here clearly $\operatorname{span}_{f}\left(P_{n}\right)=f(k-1)=\frac{k^{2}+k}{2}+1$. It is easy to show that $f$ satisfy the radio- $k$-labeling condition. As maximum color attained at $(k-1) t h$ vertex, so this radio $k$-labeling scheme will work for any path $P_{n}$ with $\frac{5 k+1}{2} \leq n \leq \frac{7 k-1}{2}$.

Example 6.2. An optimal radio 13-labeling of $P_{18}$ and radio 9-labeling of $P_{20}$ has been given in Fig. 7 and Fig. 8, respectively. 


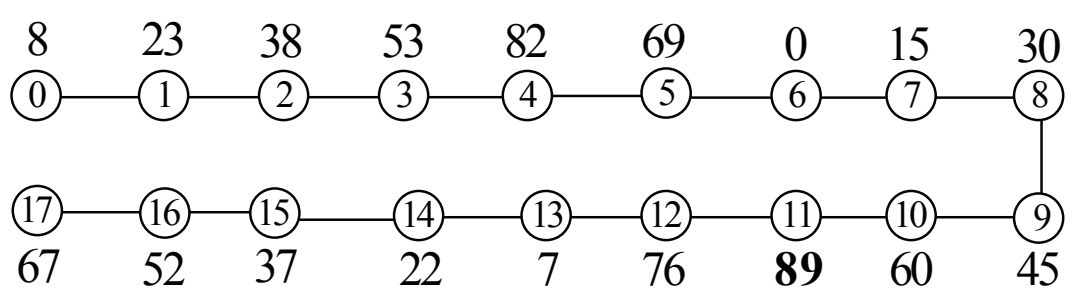

Figure 7. A radio 13-labeling of $P_{18}$ with span 89.

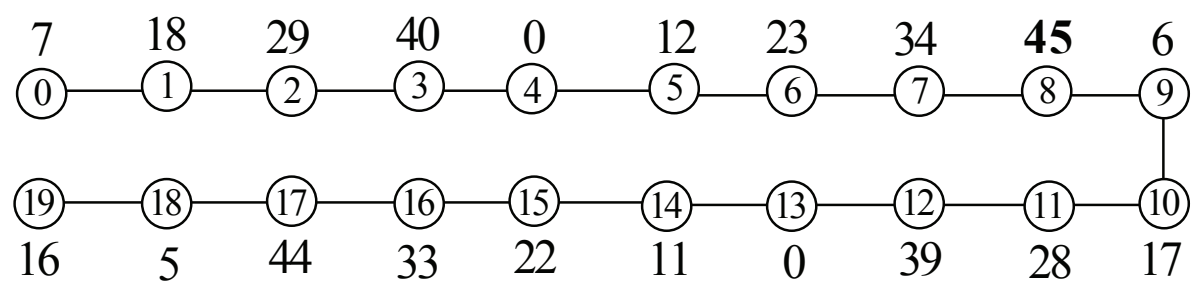

Figure 8. A radio 9-labeling of $P_{20}$ with span 45 .

\section{Concluding Remark}

Consequences of Theorem 5.1 and Theorem 6.1 include the radio $k$-chromatic number of $P_{n}$ for $k \in\{n-4, n-3, n-2, n-1\}$ (which were settled in [5,17-19,25] by different approaches). Not only that these theorem determines the radio $k$-chromatic number of $P_{n}$ for even integer $k \in\left\{\left\lceil\frac{2(n-2)}{5}\right\rceil, \ldots, n-1\right\}$ and odd integer $k \in\left\{\left\lceil\frac{2 n+1}{7}\right\rceil, \ldots, n-1\right\}$ that is at least $65 \%$ cases the radio $k$-chromatic of the path $P_{n}$ are obtained for fixed but arbitrary values of $n$. For example, if we take $n=1000$, then this article determines the exact value of $r c_{k}\left(P_{1000}\right)$ for even $k \in\{400,402, \ldots, 998\}$ and odd $k \in\{287,289, \ldots, 999\}$ where as the existing results are only for $k \in\{997,998,999\}$.

Acknowledgment. The first author is thankful to the National Board for Higher Mathematics (NBHM), India for its financial support (Grant No. 2/48(22)/R \& D II/4033). The third author is supported by the National Research Foundation of the Korean government with grant No. 2017R1D1A1B03028642.

\section{References}

[1] G.J. Chang and D. Kuo, The L(2,1)-labelling problem on graphs, SIAM J. Discrete Math. 9, 309-316, 1996.

[2] G.J. Chang, C. Lu, and S. Zhou, Distance-two labellings of Hamming graphs, Discrete Appl. Math. 157, 1896-1904, 2009.

[3] G. Chartrand, D. Erwin, and P. Zhang, Radio antipodal colorings of cycles, Proceedings of the Thirty-first Southeastern International Conference on Combinatorics, Graph Theory and Computing (Boca Raton, FL, 2000) 144, 129-141, 2000.

[4] G. Chartrand, D. Erwin, and P. Zhang, A graph labeling problem suggested by FM channel restrictions, Bull. Inst. Combin. Appl. 43, 43-57, 2005.

[5] G. Chartrand, L. Nebesky, and P. Zhang, Radio k-colorings of paths, Discuss. Math. Graph. Theory, 24, 5-21, 2004.

[6] G. Chartrand, D. Erwin, F. Harary, and P. Zhang, Radio labelings of graphs, Bull. Inst. Combin. Appl. 33, 77-85, 2001.

[7] J.P. Georges, D.W. Mauro, and M.I. Stein, Labelling products of complete graphs with a condition at distance two, SIAM J. Discrete Math. 14, 28-35, 2001. 
[8] J.R. Griggs and X.T. Jin, Real number graph labelling with distance conditions, SIAM J. Discrete Math. 20, 302-327, 2006.

[9] J.R. Griggs, and D. Král', Graph labellings with variable weights, a survey, Discrete Appl. Math. 157, 2646-2658, 2009.

[10] J.R. Griggs and R.K. Yeh, Labelling graphs with a condition at distance 2, SIAM J. Discrete Math. 5, 586-595, 1992.

[11] W.K. Hale, Frequency assignment, theory and application, Proc. IEEE, 68, 1497$1514,1980$.

[12] J.v.d. Heuvel, R. Leese, and M. Shepherd, Graph labelling and radio channel assignment, J. Graph Theory, 29, 263-283, 1998.

[13] J.S.-T. Juan and D.D.-F. Liu, Antipodal labelings for cycles, Ars Combin. 103, 81-96, 2012.

[14] M. Kchikech, R. Khennoufa, and O. Togni, Linear and cyclic radio k-labelings of trees, Discuss. Math. Graph Theory, 27 (1), 105-123, 2007.

[15] G. Chartrand, D. Erwin, F. Harary, and P. Zhang, Radio labelings of graphs, Bull. Inst. Combin. Appl. 33, 77-85, 2001.

[16] R. Khennoufa and O. Togni, The radio antipodal and radio numbers of the hypercube, Ars Combin. 102, 447-461, 2011.

[17] R. Khennoufa and O. Togni, A note on radio antipodal colourings of paths, Math. Bohem. 130 (3), 277-282, 2005.

[18] S.R. Kola and P. Panigrahi, Nearly antipodal chromatic number ac' $\left(P_{n}\right)$ of the path, Math. Bohem. 134 (1), 77-86, 2009.

[19] S.R. Kola and P. Panigrahi, On radio $(n-4)$-chromatic number of the path $P_{n}$, AKCE Int. J. Graphs Comb. 6 (1), 209-217, 2009.

[20] D. Král', The channel assignment problem with variable weights, SIAM J. Discrete Math. 20, 690-704, 2006.

[21] X. Li, V. Mak, and S. Zhou, Optimal radio labellings of complete m-ary trees, Discrete Appl. Math. 158, 507-515, 2010.

[22] D.D.-F. Liu, Radio number for trees, Discrete Math. 308, 1153-1164, 2008.

[23] D.D.-F. Liu and M. Xie, Radio number for square of cycles, Congr. Numer. 169, 105-125, 2004.

[24] D.D.-F. Liu and M. Xie, Radio number for square paths, Ars Combin. 90, 307-319, 2009.

[25] D.D.-F. Liu and X. Zhu, Multi-level distance labelings for paths and cycles, SIAM J. Discrete Math. 19, 610-621, 2005.

[26] M. Morris-Rivera, M. Tomova, C. Wyels, and Y. Yeager, The radio number of $C_{n} \times C_{n}$, Ars Combin. 103, 81-96, 2012.

[27] J.P. Ortiz, P. Martinez, M. Tomova, and C. Wyels, Radio numbers of some generalized prism graphs, Discuss. Math. Graph Theory, 31 (1), 45-62, 2011.

[28] V.S. Reddy and V.K. Iyer, Upper bounds on the radio number of some trees, Int. J. Pure Applied Math. 71 (2), 207-215, 2011.

[29] L. Saha and P. Panigrahi, A graph Radio k-coloring algorithm, Lecture notes in Computer Science 7643, 125-129, 2012.

[30] L. Saha and P. Panigrahi, On the Radio number of Toroidal grids, Australian J. Combin. 55, 273-288, 2013.

[31] L. Saha and P. Panigrahi, A lower bound for radio $k$-chromatic number, Discrete Appl. Math. 192, 87-100, 2015.

[32] U. Sarkar and A. Adhikari, On characterizing radio $k$-coloring problem by path covering problem, Discrete Math 338 (4), 615-620, 2015.

[33] P. Zhang, Radio labellings of cycles, Ars Combin 65, 21-32, 2002.

[34] S. Zhou, A channel assignment problem for optical networks modelled by Cayley graphs, Theoret. Comput. Sci. 310, 501-511, 2004. 
[35] S. Zhou, Labelling Cayley graphs on abelian groups, SIAM J. Discrete Math. 19, 985-1003, 2006.

[36] S. Zhou, A distance-labelling problem for hypercubes, Discrete Appl. Math. 156, 2846$2854,2008$. 